\title{
AVALIAÇÃO DO POTENCIAL DA TRANSPOSIÇÃO DA SERAPILHEIRA E DO BANCO DE SEMENTES DO SOLO PARA RESTAURAÇÃO FLORESTAL EM ÁREAS DEGRADADAS ${ }^{1}$
}

\author{
Bruna Dias Rodrigues², Sebastião Venâncio Martins ${ }^{3}$ e Hélio Garcia Leite ${ }^{3}$ \\ RESUMO - Este estudo teve por objetivo testar a viabilidade da transposição da serapilheira e do banco de \\ sementes do solo como metodologia de restauração florestal de áreas degradadas. Amostras de 1x1 mde serapilheira \\ e de solo superficial foram coletadas num fragmento de floresta estacional semidecidual secundária no campus \\ da Universidade Federal de Viçosa, em Viçosa, MG. As amostras de solo e de serapilheira foram depositadas \\ em canteiros, nos quais o solo superficial $(10 \mathrm{~cm})$ foi previamente retirado, no Viveiro de Pesquisas da UFV. \\ Foram comparados três tratamentos e uma testemunha com cinco repetições cada, totalizando 20 amostras. \\ O primeiro tratamento (T1) foi a transposição apenas da serapilheira, o segundo (T2) apenas do banco de \\ sementes do solo e o terceiro (T3) da serapilheira juntamente com o banco de sementes do solo. Durante \\ um período de seis meses, foram registrados 327 indivíduos de espécies arbustivo-arbóreas e 864 de espécies \\ herbáceas. Entre as espécies arbustivo-arbóreas, $74 \%$ foram pioneiras e $26 \%$ de outros grupos ecológicos. \\ A espécie arbórea mais frequente foi Cecropia hololeuca com 151 indivíduos. Entre as herbáceas, Oxalis corniculata \\ foi a mais abundante, com 329 indivíduos. As diferenças de densidade e riqueza de espécies entre os tratamentos \\ foram significativas, sendo maior riqueza obtida no tratamento T3 e maiores densidades nos tratamentos $\mathrm{T} 2$ \\ e T3. Portanto, conclui-se que a transposição do banco de sementes é uma metodologia promissora para estimular \\ a restauração florestal em áreas degradadas, sendo mais eficiente quando se utiliza o solo superficial juntamente \\ com a camada de serapilheira.
}

Palavras-chave: Nucleação, regeneração florestal e restauração ecológica.

\section{EVALUATION OF THE LITTER AND SOIL SEED BANK TRANSPOSITION AS A FOREST RESTORATION OF DEGRADED AREAS METHODOLOGY}

\begin{abstract}
This study aimed to evaluate the viability of the litter and soil seed bank transposition as a forest restoration of degraded areas methodology. The litter and soil samples of $1 \times 1 \mathrm{~m}$ were collected at a depth of $5.0 \mathrm{~cm}$ in a secondary semideciduous forest in Viçosa, MG, Brazil. The litter and soil samples were deposited in seedbeds in which the superficial soil $(10 \mathrm{~cm})$ was previously removed. Three treatments were performed as well as a separate control with five repetitions, totaling 20 samples. The first treatment (T1) was the litter transposition only, the second treatment (T2) was the soil seed bank only, the third treatment (T3) was the transposition of the litter together with the soil seed bank and the control (T). During a period of six months, 327 individuals of trees and shrubs and 864 herbaceous individuals were identified. Among the tree and shrub species occurring in the samples, $74 \%$ were classified as pioneers, and $26 \%$ were classified as other ecological groups. The most abundant tree species were Cecropia hololeuca with 151 individuals. Among the herbs, Oxalis corniculata was the most abundant, with 329 individuals. The differences of density and species richness between the treatments were significant, with the greatest richness in treatment T3 and the greatest densities in treatments T2 and T3. Therefore, it is concluded that the transposition of the seed bank is a promising methodology to stimulate forest restoration in degraded areas, being more efficient when the superficial soil is used with the litter.
\end{abstract}

Keywords: Nucleation, forest regeneration and ecological restoration.

\footnotetext{
${ }^{1}$ Recebido em 15.05.2009 e aceito para publicação em 14.10.2009.

${ }^{2}$ Multigeo Engenharia Mineral Geologia e Meio Ambiente, Avenida Francisco Sá, 1213 - sala 1301, CEP 30441-021, Belo Horizonte, MG. E-mail: < brubsrodrigues@yahoo.com.br>.

${ }^{3}$ Departamento de Engenharia Floresta, Universidade Federal de Viçosa - Vicosa, MG - Brasil. E-mail: <venancio@ufv.br> e<hgleite@gmail.com>.
} 


\section{INTRODUÇÃO}

Nas duas ultimas décadas tem aumentado o interesse de pesquisadores e empresas dos setores de mineração, geração de energia e florestal na busca de novas alternativas de recuperação de áreas degradadas num contexto ecológico. Assim, a recuperação ambiental tem enfatizando a recomposição da diversidade de espécies e a sustentabilidade dos ecossistemas recuperados, o que vem sendo tratado como restauração ecológica e, no caso especifico de ecossistemas florestais, de restauração florestal. Esta nova tendência da recuperação de áreas degradadas tem sido amplamente abordada em recentes publicações no Brasil e no exterior (CHOI, Y.D., 2004; PETERSEN e CLARK, J.J., 2005; MARTINS, 2007, 2009a e b; MARTINS et al., 2007, 2009; VIEIRA, D.L.M. e SCARIOT, A. 2006; RODRIGUES et al., 2004, 2007).

Um dos objetivos das técnicas de restauração florestal é o aproveitamento do potencial de resiliência (auto-regeneração) das áreas a serem recuperadas e da máxima quantidade e diversidade de material vegetal (propágulos e restos vegetais) disponível em áreas cujo licenciamento ambiental para atividades de mineração, represamento de cursos d'água e outros permite que a vegetação seja suprimida (MARTINS et al, 2007, MARTINS, 2009a).

A serapilheira é particularmente importante por atuar na superfície do solo como um sistema de entrada e saída, recebendo entradas via vegetação e, por sua vez, decompondo-se e suprindo o solo e as raízes com nutrientes e matéria orgânica, sendo essencial na restauração da fertilidade do solo em áreas em início de sucessão ecológica (EWEL, 1976). Além disto, funciona como uma manta que facilita a entrada de sementes e sua incorporação ao banco de sementes do solo.

O banco de sementes é composto pelas sementes viáveis, em estado de dormência real ou imposta, presentes na superfície ou no interior do solo de uma determinada área (HARPER, 1977). Esse componente florestal está diretamente ligado ao estabelecimento de populações de plantas, à manutenção da diversidade de espécies, ao estabelecimento de grupos ecológicos e à restauração da riqueza de espécies durante a regeneração da floresta após distúrbios naturais ou antrópicos (HARPER, 1977; ZHANG et al., 2001). Por isso, tem sido utilizado como indicador ecológico de avaliação e monitoramento da regeneração de ecossistemas em restauração (BRAGA et al., 2008; MARTINS, et al., 2008; MARTINS, 2009a e b).

A transposição da serapilheira e do banco de sementes do solo pode ser uma alternativa viável para acelerar o processo de sucessão em áreas em que o solo foi degradado (MARTINS, 2007, 2009b; MARTINS et al., 2009). Na camada de restos vegetais e de solo superficial de uma floresta além de sementes de espécies de diferentes formas de vida, são encontrados ainda nutrientes, matéria orgânica e microrganismos, essenciais para a recuperação da fertilidade e da atividade biológica destes solos). Contudo, ainda não existe na literatura especializada uma definição de qual o melhor componente do banco de sementes - serapilheira ou solo superficial - deve ser transposto visando estimular a sucessão em solos degradados, dadas às diferenças em suas composições e densidades de sementes.

Partindo-se da hipótese de que existem diferenças de densidade e riqueza de espécies no estoque de sementes da camada de serapilheira e de solo superficial de uma floresta, este estudo teve por objetivos comparar a viabilidade da transposição destes dois componentes como metodologia de estímulo da restauração florestal.

\section{MATERIAL E MÉTODOS}

\subsection{Caracterização da área de estudo}

O estudo foi realizado no município de Viçosa ( $20^{\circ} 45^{\prime} \mathrm{S}$ e $42^{\circ} 51^{\prime} \mathrm{W}$ ), na mesorregião da Zona da Mata mineira. A coleta da serapilheira e do banco de sementes do solo foi realizada em um fragmento de Floresta Estacional Semidecidual (VELOSO et al., 1991) secundária localizado no campus da Universidade Federal de Viçosa, conhecido por "Mata da Agronomia". Este fragmento encontra-se em estádio médio de sucessão florestal com predominância de espécies secundárias iniciais típicas da região e pioneiras nas bordas. E por estar dentro do campus da UFV, vem sendo bem conservado nas ultimas duas décadas, sem nenhum tipo de perturbação antrópica ou entrada de espécies invasoras.

O clima da região de Viçosa é do tipo Cwb, segundo a classificação de Köppen, ou seja, mesotérmico com verões quentes e chuvosos e invernos frios e secos. A temperatura média anual é de $21,8^{\circ} \mathrm{C}$ e a precipitação pluviométrica anual de $1314.2 \mathrm{~mm}$ (CASTRO et al., 1983). 
O relevo é acidentado, fazendo parte do chamado domínio do "Mar de Morros" com predomínio de duas classes de solos. Nos topos de morro e encostas predomina o Latossolo Vermelho-Amarelo álico, enquanto nos terraços predomina o Podzólico Vermelho-Amarelo câmbico fase terraço (CORREA, 1984). As altitudes variam de 690 a 800 m (BRAZ et al., 2002).

\subsection{Transposição da serapilheira e do banco de sementes do solo}

Para avaliar a composição florística e a densidade das espécies oriundas da transposição do banco de sementes do solo e da serapilheira foram coletadas amostras superficiais do solo ( $5 \mathrm{~cm}$ de profundidade) e da serapilheira presentes na área de estudo. Esta amostragem da camada superficial do solo $(5 \mathrm{~cm})$ tem sido a comumente utilizada em estudos desta natureza por ser a mais representativa em termos de densidade e riqueza de sementes viáveis do banco (BRAGA et al., 2008; MARTINS, S.V. et al., 2008; MARTINS, 2009b).

Foram retiradas 10 amostras do banco de sementes do solo e 10 amostras de serapilheira com o auxílio de um gabarito (moldura de madeira) com a dimensão de 1 x 1m (1,0 m²), lançado de forma aleatória no chão da floresta, ou seja, sem um espaçamento pré-definido entre as amostragens, contudo mantendo-se uma distância de $50 \mathrm{~m}$ da borda do fragmento.

O material coletado foi colocado em sacos plásticos identificados e em seguida transportado para o viveiro de pesquisa da Universidade Federal de Viçosa.

Foram analisados três tratamentos e uma testemunha, com cinco repetições cada, totalizando 20 amostras. O primeiro tratamento (T1) foi a transposição da serapilheira apenas, o segundo tratamento (T2) a transposição do banco de sementes do solo, o terceiro tratamento (T3) a transposição da serapilheira juntamente com o banco de sementes do solo e a testemunha $(\mathrm{T})$.

Para evitar contaminação com sementes oriundas das proximidades, todo o viveiro foi mantido limpo e sem vegetação ruderal durante todo o período do experimento. Mas para simular uma situação real de transposição do banco de sementes para uma área degradada, não foi utilizado nenhum tipo de cobertura dos canteiros.
Cada amostra de solo e de serapilheira foi depositada em um canteiro de $1 \times 1 \mathrm{~m}$ delimitado no viveiro por blocos de concreto no qual o solo superficial foi removido até uma profundidade de $10 \mathrm{~cm}$. Além da remoção do solo superficial dos canteiros e, portanto, do banco de sementes que por ventura poderia haver, toda vegetação herbácea encontrada no viveiro foi removida para evitar contaminações. A distribuição das amostras nos canteiros seguiu o delineamento inteiramente casualizado (DIC).

Como o experimento foi montado na estação seca, foi necessária a irrigação diária dos canteiros. Foi realizado acompanhamento da germinação das sementes ao longo de seis meses, durante o período de maio a novembro de 2008 .

A avaliação do experimento consistiu na quantificação do número de plântulas que emergiram da serapilheira e do banco de sementes do solo. Todos os indivíduos que germinaram foram identificados utilizando-se literatura especializada, consulta a herbários e especialistas; sendo classificados quanto à forma de vida em espécies arbóreas, arbustivas e herbáceas. As espécies foram também classificadas em grupos ecológicos, com base nos trabalhos de Silva et al. (2003), Martins et al. (2004) e Martins (2007).

\section{RESULTADOS}

Da avaliação das plântulas germinadas nos tratamentos, foram identificados 327 indivíduos arbóreos e arbustivos e 864 indivíduos herbáceos (Tabelas 1 e 2).

Quanto às formas de vida das espécies germinadas, a herbácea foi predominante, seguida da arbórea e da arbustiva. Dentre as espécies arbóreas e arbustivas ocorrentes nas amostras, $74 \%$ foram classificadas como pioneiras e $26 \%$ como outros grupos ecológicos.

A espécie arbórea mais frequente nos tratamentos foi Cecropia hololeuca com 151 indivíduos ou 46,17\% do total amostrado entre as espécies arbóreas. Cecropia hololeuca, Croton urucurana (43 indivíduos) e Solanum erianthum (33 indivíduos) representam 69,41\% dos indivíduos arbustivo-arbóreos identificados nas amostras.

Em relação às herbáceas, Oxalis corniculata foi a mais numerosa, com 329 indivíduos. As três espécies mais numerosas de herbáceas foram: Oxalis corniculata, Diodia brasiliensis (91 indivíduos) e Cyperus esculentus (89 indivíduos).

R. Árvore, Viçosa-MG, v.34, n.1, p.65-73, 2010 
Tabela 1 - Espécies herbáceas e respectivos números de indivíduos germinados nos tratamentos (T, T1, T2, T3). Transposição da serapilheira e do banco de sementes do solo, Viçosa, MG.

Table 1- Herbaceous species and their number of individuals germinated in the treatments (T, T1, T2, T3). Litter and soil seed bank transposition, Viçosa, $M G$.

\begin{tabular}{|c|c|c|c|c|c|}
\hline Espécie & Família & $\mathrm{T}$ & $\mathrm{T} 1$ & $\mathrm{~T} 2$ & $\overline{\mathrm{T} 3}$ \\
\hline Ruellia brevifolia $\mathrm{L}$. & Acanthaceae & & 1 & 1 & \\
\hline Amaranthus deflexus L. & Amaranthaceae & & 4 & 4 & 2 \\
\hline Amaranthus hybridus var. paniculatus (L.) Thell. & Amaranthaceae & & & 1 & \\
\hline Conium maculatum $\mathrm{L}$. & Apiaceae & 14 & & 5 & \\
\hline Spilanthes acmella (L.) Murray & Asteraceae & & 2 & & \\
\hline Baccharis dracunculifolia DC. & Asteraceae & & & 2 & 1 \\
\hline Siegesbeckia orientalis L. & Asteraceae & & & 2 & \\
\hline Erechtites hieraciifolius (L.) Raf. ex DC. & Asteraceae & & 1 & & 2 \\
\hline Sonchus oleraceus L. & Asteraceae & 16 & 5 & 4 & 9 \\
\hline Emilia sonchifolia (L.) DC. & Asteraceae & 12 & 5 & 17 & 2 \\
\hline Impatiens walleriana Hook. f. & Balsaminaceae & & & 1 & \\
\hline Lepidium virginicum $\mathrm{L}$. & Brassicaceae & & & 2 & \\
\hline Ipomoea batatoides Choisy & Convolvulaceae & & 1 & 1 & \\
\hline Cyperus rotundus $\mathrm{L}$. & Cyperaceae & & 28 & & 7 \\
\hline Cyperus esculentus L. & Cyperaceae & & 7 & 80 & 2 \\
\hline Buddleja brasiliensis Jacq. ex Spreng. & Loganiaceae & 48 & 2 & 10 & \\
\hline Oxalis corniculata $\mathrm{L}$. & Oxalidaceae & 104 & 61 & 105 & 59 \\
\hline Phyllanthus tenellus Roxb. & Phyllanthaceae & & 3 & 7 & 3 \\
\hline Plantago tomentosa $\mathrm{L}$. & Plantaginaceae & 2 & & 1 & \\
\hline Urochloa decumbens (Stapf) R.D. Webster & Poaceae & 23 & 2 & 16 & 26 \\
\hline Panicum maximum Jacq. & Poaceae & & 3 & 16 & 2 \\
\hline Eleusine indica (L.) Gaertn. & Poaceae & 2 & & 3 & \\
\hline Portulaca oleracea L. & Portulacaceae & & & 1 & \\
\hline Diodia brasiliensis Spreng. & Rubiaceae & & 8 & 79 & 4 \\
\hline Solanum americanum Mill. & Solanaceae & & 1 & 22 & 7 \\
\hline Solanum sisymbrifolium Lam. & Solanaceae & & & 2 & 1 \\
\hline Total & & 221 & 134 & 382 & 127 \\
\hline
\end{tabular}

\section{Densidade}

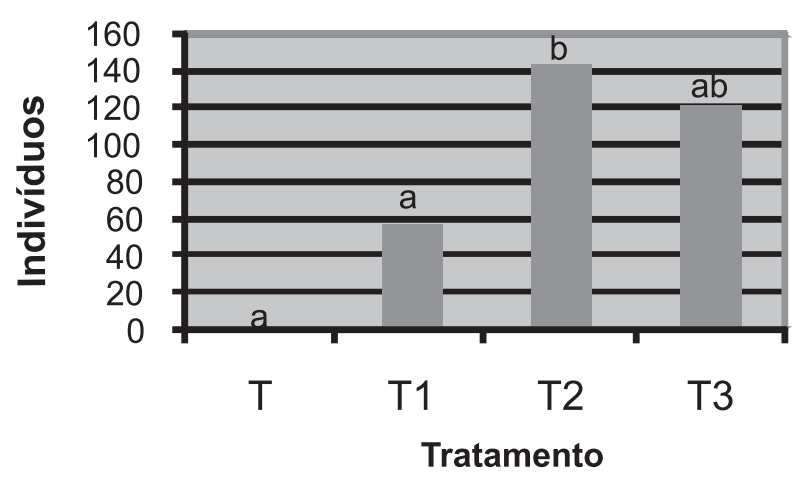

Figura 1 - Densidade de indivíduos arbustivo-arbóreos em todos os tratamentos. Transposição da serapilheira e do banco de sementes do solo, Viçosa, MG. Barras seguidas pelas mesmas letras não diferem entre si a $5 \%$.

Figure 1-Density of shrub and tree individuals in all treatments. Litter and soil seed bank transposition, Viçosa, MG. Bars followed by the same letter do not differ between themselves $5 \%$.

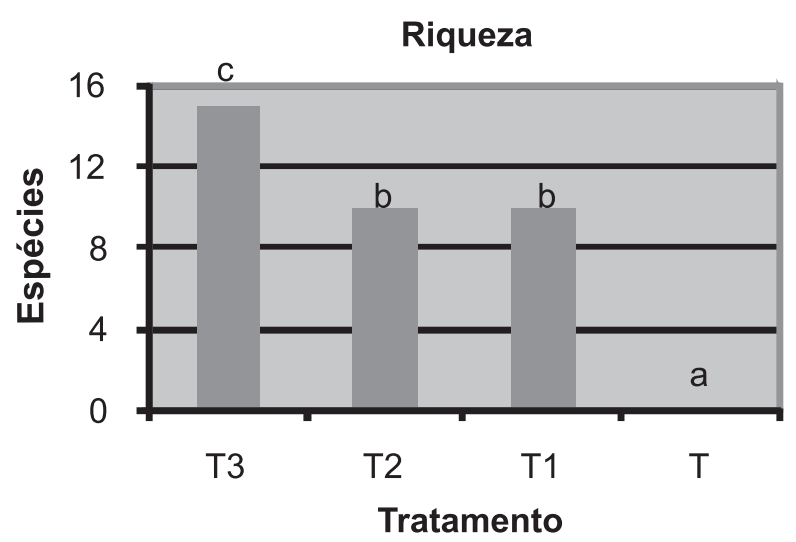

Figura 2 - Riqueza de espécies arbustivo-arbóreas em todos os tratamentos. Transposição da serapilheira e do banco de sementes do solo, Viçosa, MG. Barras seguidas pelas mesmas letras não diferem entre si a $5 \%$.

Figure 2 - Abundance of shrub and tree species in all treatments. Litter and soil seed bank transposition, Viçosa, MG. Bars followed by the same letters do not differ between themselves $5 \%$. 
Tabela 2 - Espécies arbustivo-arbóreas e respectivos grupos ecológicos e números de indivíduos germinados nos tratamentos (T, T1, T2, T3). Transposição da serapilheira e do banco de sementes do solo, Viçosa, MG. P = espécie pioneira; $\mathrm{St}=$ espécie secundária tardia; $\mathrm{Si}=$ espécie secundária inicial; $\mathrm{Sc}=$ espécie não classificada.

Table 2 - Tree and shrub species and their ecological group and number of individuals germinated in the treatments (T, T1, T2, T3). Litter and soil seed bank transposition, Viçosa, MG. P = pioneer species; $S t=$ secondary late species; $\mathrm{Si}=$ secondary early species; and $\mathrm{Sc}=$ not classified .

\begin{tabular}{|c|c|c|c|c|c|c|}
\hline Espécie & Família & GE & $\mathrm{T} 1$ & $\mathrm{~T} 2$ & T3 & $\mathrm{T}$ \\
\hline Pfaffia glomerata (Spreng.) Pedersen & Amaranthaceae & $\mathrm{Sc}$ & 3 & 7 & 5 & \\
\hline Piptocarpha macropoda (DC.) Baker & Asteraceae & $\mathrm{P}$ & & 18 & 1 & \\
\hline Trema micrantha (L.) Blume & Cannabaceae & $\mathrm{P}$ & 2 & 18 & 8 & \\
\hline Carica papaya $\mathrm{L}$. & Caricaceae & Sc & 1 & & & \\
\hline Erythroxylum tortuosum Mart. & Erythroxylaceae & $\mathrm{St}$ & & & 3 & \\
\hline Croton urucurana Baill. & Euphorbiaceae & $\mathrm{P}$ & & 22 & 21 & \\
\hline Apuleia leiocarpa (Vogel) J.F. Macbr. & Fabaceae - Caesalpinioideae & $\mathrm{Si}$ & & & 2 & \\
\hline $\begin{array}{l}\text { Piptadenia gonoacantha (Mart.) } \\
\text { J.F. Macbr. }\end{array}$ & Fabaceae - Mimosoideae & $\mathrm{Si}$ & 1 & & & \\
\hline $\begin{array}{l}\text { Pseudopiptadenia contorta (DC.) } \\
\text { G.P. Lewis \& M.P. Lima }\end{array}$ & Fabaceae - Mimosoideae & $\mathrm{Si}$ & & 2 & & \\
\hline Aegiphila sellowiana Cham. & Lamiaceae & $\mathrm{P}$ & 4 & 2 & 4 & \\
\hline Luehea grandiflora Mart. & Malvaceae & $\mathrm{Si}$ & & & 1 & \\
\hline Miconia cinnamomifolia (DC.) Naudin & Melastomataceae & $\mathrm{Si}$ & & & 1 & \\
\hline Eucalyptus citriodora Hook. & Myrtaceae & $\mathrm{Sc}$ & & & 1 & \\
\hline Zanthoxylum rhoifolium Lam. & Rutaceae & $\mathrm{Si}$ & & & 1 & \\
\hline Solanum cernuиm Vell. & Solanaceae & $\mathrm{P}$ & 2 & 2 & 4 & \\
\hline Solanum erianthum D. Don & Solanaceae & $\mathrm{P}$ & 10 & 4 & 19 & \\
\hline Solanum paniculatum L. & Solanaceae & $\mathrm{P}$ & 2 & & 1 & \\
\hline Vassobia breviflora (Sendtn.) Hunz. & Solanaceae & $\mathrm{P}$ & 1 & 3 & & \\
\hline Cecropia hololeuca Miq. & Urticaceae & $\mathrm{P}$ & 31 & 69 & 51 & \\
\hline Total & & & 57 & 147 & 123 & \\
\hline
\end{tabular}

Nas repetições da testemunha (T) houve germinação apenas de espécies herbáceas, com nenhuma germinação de espécies arbóreas ou arbustivas.

Em relação às espécies arbustivo-arbóreas, o tratamento 2 proporcionou maior número de indivíduos em comparação com os demais tratamentos; enquanto o tratamento 3 obteve maior número de espécies em relação aos outros tratamentos (Figuras 1 e 2). Estes resultados foram analisados estatisticamente por meio de uma Análise de Variância e constatou-se que a $1 \%$ de probabilidade as variáveis densidade e riqueza foram significativas, ou seja, apresentaram diferença entre os tratamentos avaliados. Posteriormente foi efetuado o teste de Tukey a $5 \%$ de probabilidade e constatou-se que para a variável densidade, o tratamento 2 e o tratamento 3 foram estatisticamente iguais. Já em relação à variável riqueza o teste constatou que o tratamento 1 e o tratamento 2 foram estatisticamente iguais e diferiram da testemunha e do tratamento 3 , que obteve maior média em relação aos demais.

\section{DISCUSSÃO}

A presença de diversas categorias sucessionais observadas no presente estudo pode estar associada à deciduidade parcial presente nas Florestas Estacionais Semideciduais que propiciam diferentes microambientes para que diversas espécies cresçam em diferentes situações.

O destaque em densidade de espécies herbáceas em todos os tratamentos pode ser atribuído à participação delas no banco de sementes do solo e na serapilheira da floresta onde as amostras foram coletadas, já que se trata de um fragmento florestal cujo entorno é formado por experimentos de agricultura, mas também porque estes tratamentos favoreceram o recrutamento dessas plantas, por apresentarem condições de fertilidade do solo melhores para germinação das mesmas. Cabe destacar que a presença dessas espécies herbáceas também na testemunha mostra que certo nível de contaminação ocorreu, mas apenas para este grupo de plantas uma vez que não foram encontradas espécies arbustivas e arbóreas nas parcelas sem transposição do banco. 
Segundo Hopkins et al. (1990), a situação de dominância das espécies herbáceas é comum em trabalhos com banco de sementes de comunidades florestais fragmentadas e cercadas de vegetação antropizada (pastos, culturas agrícolas, etc.). Alguns fatores, como mecanismos eficientes de dispersão, tamanho e dormência das sementes destas espécies colaboram para este padrão (GARWOOD, 1989).

Nesse estudo, ficou evidente que a serapilheira teve um efeito desejável em inibir à germinação dessas espécies herbáceas, já que nos tratamentos em que ela foi utilizada (T1 e T3) o número de plântulas deste grupo germinadas foi consideravelmente menor (134 em T1 e 127 em T3) do que no tratamento utilizando apenas o banco de sementes do solo (382 em T2). Portanto, a camada de serapilheira deve ter funcionado como uma barreira dificultando o recrutamento de espécies herbáceas do banco de sementes. Como espécies herbáceas possuem em geral sementes muito pequenas, que podem penetrar a camada de serapilheira até atingir o solo, esta poderia atuar como barreira física dificultando a passagem de luz para estimular a germinação destas sementes e a emergência das plântulas que eventualmente conseguissem germinar. Este efeito é desejável, uma vez que, a abundância de espécies herbáceas pode dificultar a regeneração de espécies arbustivo-arbóreas responsáveis pela rápida sucessão secundária. No tratamento T2, em que o solo superficial foi depositado sem cobertura de serapilheira, a exposição à luz favoreceu a germinação e estabelecimento de sementes de ervas.

Estes resultados corroboram estudos que demonstram que a serapilheira pode ter um efeito fortemente inibitório sobre a germinação e emergência, sendo as espécies de menores sementes as mais afetadas (BERGELSON, 1991; MOLOFSKY eAUGSPURGUER, 1992; VASQUEZ-YANES e OROZCO-SEGOVIA, 1992; DALLING et al., 1998; SINGHAKUMARA, 2000).

O tratamento 3 (serapilheira + banco de sementes do solo) mesmo sendo estatisticamente igual ao tratamento 2 (banco de sementes do solo) em relação à densidade de espécies arbustivo-arbóreas, proporcionou maior riqueza de espécies em relação aos demais tratamentos. Cabe destacar que sementes maiores de espécies arbóreas podem permanecer na serapilheira por longo tempo antes de serem incorporadas ao solo (CHAMBERS e MACMAHON, 1994), sendo este também um importante reservatório de sementes grandes. Além disso, a presença da serapilheira é uma fonte de matéria orgânica e nutrientes para as sementes viáveis do banco, além de aumentar a retenção da água de chuva e cedêla lentamente ao solo, aumentando a disponibilidade de água principalmente durante a estação seca, o que pode contribuir para uma germinação mais abundante e prolongada durante o ano. Convém lembrar também que esse "efeito esponja" da serapilheira potencializa a infiltração de água no solo, em detrimento do escoamento superficial, colaborando substancialmente para conservação e proteção do solo transposto, bem como do solo como um todo, especialmente em áreas declivosas; além de reduzir a amplitude térmica do solo e consequentemente a evaporação de água. Logo, a transposição do conjunto solo e serapilheira é a mais indicada para a recuperação de áreas em que o solo foi degradado.

Os padrões de deposição da serapilheira produzem heterogeneidade temporal e espacial no ambiente florestal, podendo afetar a estrutura e dinâmica da comunidade de plantas (FACELLI e PICKETT, 1991; MOLOFSKY e AUGSPURGER, 1992) e, portanto, este componente constitui um aspecto relevante para o conhecimento e preservação dos ecossistemas naturais.

O papel da transposição do banco de sementes em estimular a nucleação em solo sem vegetação ficou evidenciado também pelo resultado encontrado nas parcelas testemunhas que apresentaram a menor densidade de germinação de plântulas em geral mantendo o solo praticamente exposto, sendo esta extremamente baixa germinação resultante apenas de espécies herbáceas, uma vez que não foi observada germinação de espécies arbustivo-arbóreas.

Apesar da expressiva presença de espécies herbáceas no banco de sementes, a alta densidade de espécies arbustivo-arbóreas como Cecropia hololeuca, Croton urucurana e Solanum erianthum refletem o potencial do uso da transposição do conjunto solo e serapilheira em promover a nucleação em áreas degradadas.

Estudo sobre caracterização do banco de sementes o solo de um fragmento florestal na Zona da Mata de Minas Gerais, também apontou $C$. hololeuca como uma das espécies arbóreas mais abundantes, com 46,64\% do total de indivíduos amostrados (BRAGA et al., 2008). 
Por se tratar de espécie pioneira de rápido crescimento em clareiras, bordas de matas, áreas abertas em geral, solos pobres e ácidos e com frutificação atrativa a pássaros e morcegos (GANDOLFI, 2000; MARTINS, 2007), a abundância de $C$. hololeuca no banco de sementes do solo lhe confere importante papel em estimular a sucessão florestal em áreas degradadas, o que também reforça a viabilidade da transposição do banco de sementes como metodologia de restauração florestal. Grandes áreas abertas com solo exposto, como áreas degradadas por atividades de mineração, por exemplo, apresentam como característica oscilação de temperaturas diurnas e noturnas e elevados níveis de luz na superfície do solo, fatores essenciais para a germinação de $C$. hololeuca (GODOI e TAKAKI, 2004).

Das espécies arbóreas e arbustivas presentes no material coletado, $76 \%$ são espécies pioneiras. O destaque de espécies pioneiras no banco de sementes do solo deve-se a capacidade de formarem banco persistente devido à longa viabilidade das sementes, a grande produção de sementes e a eficientes mecanismos de dispersão (DALLING, 2002). Em contrapartida, espécies típicas de estádios mais avançados da sucessão apresentam sementes recalcitrantes, as quais são geralmente grandes, com elevadas taxas de metabolismo e curta viabilidade. Este fato, contudo, não compromete a técnica de transposição do banco de sementes visando recuperar áreas degradadas, uma vez que as espécies pioneiras conseguindo se estabelecer passam a favorecer a entrada posterior de espécies secundárias tardias, contribuindo para o avanço da sucessão ecológica nestas áreas. Além disto, após o estabelecimento das espécies pioneiras via transposição do banco de sementes, a comunidade em restauração pode ser enriquecida através de semeadura direta de espécies secundárias tardias.

\section{CONCLUSÕES}

A transposição do banco de sementes configurase como uma alternativa viável para estimular a sucessão florestal em áreas degradadas, sendo que melhores resultados em termos de densidade e riqueza de espécies são obtidos quando solo superficial e camada de serapilheira são utilizados em conjunto. Espécies arbóreas pioneiras abundantes no banco de sementes têm sua germinação estimulada quando transferidas para áreas abertas e solos expostos, podendo colonizar rapidamente estas áreas.

\section{REFERÊNCIAS}

BERGELSON, J. Competition between plants, before and after death. Tree, v.112, p.378-379, 1991.

BRAGA, A. J. T. et al. Composição do banco de sementes de uma floresta semidecidual secundária considerando o seu potencial de uso para recuperação ambiental. Revista Árvore, v.32, n.6, p.1089-1098, 2008.

BRAZ, D. M.; CARVALHO-OKANO, R. M.; KAMEYAMA. C. Acanthaceae da Reserva Florestal Mata do Paraíso, Viçosa, Minas Gerais. Revista Brasileira de Botânica, v.25, n.4, p.495-504, 2002.

CASTRO, P. S. et al. Interceptação da chuva por mata natural secundária na região de Viçosa, MG. Revista Árvore, v.7, n.1, p.76-89, 1983.

CHAMBERS, J. C.; MACMAHON, J.A. A day in the life of a seed: movements and fates of seeds and their implications for natural and managed systems. Annual Review of Ecology and Systematics, v. 25, p.263292, 1994.

CHOI, Y. D. Theories for ecological restoration in changing environment: toward "futuristic" restoration. Ecological Research, v.19, n.1, p.75-81, 2004.

CORREA, G. F. Modelo de evolução e mineralogia da fração argila de solos do Planalto de Viçosa, MG. 1983. 83f. Dissertação (Mestrado em Solos e Nutrição de Plantas) - Universidade Federal de Viçosa, Viçosa, MG, 1984.

DALLING, J. W.; HUBBELL, S. P.; SILVERA, K. Seed dispersal, seedling establishment and gap partitioning among tropical pioneer trees. Journal of Ecology, v.86, p.674-689, 1998.

DALling, J. W. Ecologia de semillas. In: GUARIGUATA, M. R.; KATTAN, G. H. (Eds.) Ecología y conservación de bosques neotropicales. Cartago: Ediciones LUR, 2002. p.345-375.

EWEL, J. J. Litter fall and leaf decomposition in a tropical forest succession in eastern Guatemala. Journal of Ecology, v.64, n.1, p.293-308, 1976.

R. Árvore, Viçosa-MG, v.34, n.1, p.65-73, 2010 
FACELLI, J. M.; PICKETT, S. T. A. Plant litter: its dynamics and effects on plant community structure. The Botanical Review, v.57, n.1, p.1-32, 1991.

GARWOOD, N. C. Tropical soil seeds banks: a review. In: LECK, M.; PARKER, V.; SIMPSON, R. (Eds.). Ecology of soil seed banks. San Diego: Academic, 1989. p.149-209.

GODOI, S.; TAKAKI, M. Effects of light and temperature on seed germination in Cecropia hololeuca Miq. (Cecropiaceae). Brazilian Archives of Biology and Technology, v.47, n.2, p.185-191, 2004.

HARPER, J. L. Population biology of plants. London, Academic Press, 1977. 892p.

HOPKINS, M. S.;TRACEY, J. G.; GRAHAM, A. W. The size and composition of soil seed banks in remmant patches of three structural rainforest types in North Queensland, Australia.

Australian Journal of Ecology, v. 15, n1, p. 43-50, 1990.

MARTINS, S. V. Recuperação de matas ciliares. Viçosa, MG: Aprenda Fácil, 2007. 255p.

MARTINS, S. V. Recuperação de áreas degradadas: ações em Áreas de Preservação Permanente, voçorocas, taludes rodoviários e de mineração. Viçosa, MG: Aprenda Fácil, 2009a. 270p.

MARTINS, S. V. et al. Colonization of gaps produced by death of bamboo clumps in a semideciduous mesophytic forest in southeastern Brazil. Plant Ecology, v.172, p.121$131,2004$.

MARTINS, S. V. et al. A contribuição da ecologia florestal no desenvolvimento de modelos e técnicas de restauração florestal de áreas degradadas. Revista Ação Ambiental, v.10, n.36, p.10-13, 2007.

MARTINS, S. V. et al. Banco de sementes como indicador de restauração de uma área degradada por mineração de caulim em Brás Pires, MG. Revista Árvore, v.32, n.6, p.1081-1088, 2008.

R. Árvore, Viçosa-MG, v.34, n.1, p.65-73, 2010
MARTINS, S. V. Soil seed bank as indicator of forest regeneration potential in canopy gaps of a semideciduous forest in Southeastern Brazil. In: FOURNIER, M. V. (Ed.) Forest regeneration: ecology, management and economics. New York: Nova Science Publishers, 2009b. p.113-128

MARTINS, S. V. et al. Sucessão ecológica: fundamentos e aplicações na restauração de ecossistemas florestais. In: MARTINS, S. V. (Ed.) Ecologia de florestas tropicais do Brasil. Viçosa, MG: Universidade Federal de Viçosa, 2009a. p.19-51.

MOLOFSKY, J.; AUGSPURGUER, C. K. The effect of leaf litter on early seedling establishment in a tropical Forest. Ecology, v.73, n.1, p.68-77, 1992.

RODRIGUES, R. R.; MARTINS, S. V.; BARROS, L. C. Tropical Rain Forest regeneration in an area degraded by mining in Mato Grosso State, Brazil. Forest Ecology and Management, v.190, p.323-333, 2004.

RODRIGUES, R. R.; MARTINS, S. V.; GANDOLFI, $S$. High diversity forest restoration in degraded areas: methods and projects in Brazil. New York: Science Publishers, 2007. 286p.

SILVA, A. F. et al. Composição florística e grupos ecológicos das espécies de um trecho de floresta semidecídua submontana da fazenda São Geraldo, Viçosa-MG. Revista Árvore, v.27, n.3, p.311319, 2003.

SINGHAKUMARA, B. M. P.; UDOPORUWA, R. S. J. P.; ASHTON, P. M. S. Soil seed banks in relation to light and topographic position of a hill Dipterocarp forest in Sri Lanka. Biotropica, v.32, n.1, p.190-196, 2000.

VÁSQUEZ-YANES, C.; OROZCO-SEGOVIA, A. Effect of litter from a tropical rain Forest on tree seed germination and estlablishment under controlled conditions. Tree Physiology, v.11; p.391-400, 1992.

VELOSO, H. P.; RANGEL FILHO, A. L. R.; LIMA, J. C. A classificação da vegetação brasileira, adaptada a um sistema universal. Rio de Janeiro: IBGE, 1991.123p. 
VIEIRA, D. L. M.; SCARIOT, A. Principles of natural regeneration of tropical dry forest for restoration. Restoration Ecology, v.14, n.1, p.11-20, 2006.
ZHANG, Z. Q. et al. Soil seed banks as an input of seed source in revegetation of lead/zinc mine tailings. Restoration Ecology, v.9, p.378-385, 2001. 
